\title{
Protective Effect of All-Trans Retinoic Acid in Cisplatin-Induced Testicular Damage in Rats
}

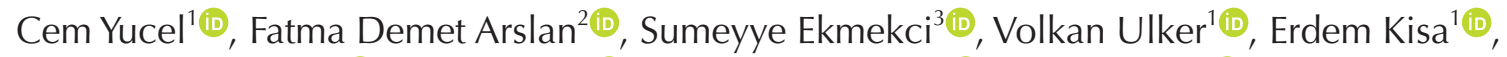 \\ Elcin Erdogan Yucel ${ }^{4}$ (i) Murat Ucar $^{1}$,, Yusuf Ozlem Ilbey ${ }^{1}$, Orcun Celik $^{1}$ (D), Banu Isbilen Basok $^{2}$ (i), \\ Zafer Kozacioglu ${ }^{1}$ iD \\ Departments of ${ }^{1}$ Urology, ${ }^{2}$ Medical Biochemistry, ${ }^{3}$ Pathology, and ${ }^{4}$ Internal Medicine, Tepecik Training and Research Hospital, Izmir, Turkey
}

Purpose: To investigate the effects of all-trans retinoic acid (ATRA) in cisplatin (CP)-induced testicular damage in rats.

Materials and Methods: Twenty-eight male Wistar rats were divided into four groups: Control, ATRA alone, ATRA+CP, and CP alone. Body weight, testicular weight, sperm count, sperm motility, percentage of abnormal sperm, total antioxidant status (TAS), total oxidant status (TOS), oxidative stress index (OSI) in testicular tissue, and testicular histopathology were compared among groups.

Results: The sperm count and motility significantly decreased and the percentage of abnormal sperm significantly increased in the CP group compared to the control and ATRA groups. CP+ATRA administration significantly increased the sperm count and motility, but reduced the abnormal sperm count. CP administration significantly increased TOS and OSI compared to the control group and the other groups. Administering CP+ATRA significantly decreased TOS and the OSI in testicular tissue and reduced spermatogenesis, but increased the Johnsen score.

Conclusions: The destructive effects of $\mathrm{CP}$ treatment on testicular tissue and spermatogenesis were reduced by administering ATRA.

Keywords: Cisplatin; Oxidative stress; Spermatogenesis; Testis; Vitamin A

This is an Open Access article distributed under the terms of the Creative Commons Attribution Non-Commercial License (http://creativecommons.org/licenses/by-nc/4.0) which permits unrestricted non-commercial use, distribution, and reproduction in any medium, provided the original work is properly cited.

\section{INTRODUCTION}

Cisplatin (CP) is an effective antineoplastic agent used to treat lung cancer and lymphoma, as well as many solid tumors such as those of the testis, stomach, ovaries, and cervix [1]. It has anti-cancer effects by inducing apoptosis through DNA alkylation [2]. Many side effects are caused due to its cytotoxicity, of which testicular toxicity is among the most severe. Therefore, its use may be restricted.

Although the cellular mechanism of CP-induced testicular toxicity is not fully understood, tissue damage induced by free radicals and reactive oxygen species (ROS) are a potential cause of CP-induced testicular toxicity [3,4]. ROS lead to apoptosis through cellular damage [5]. They are also involved in aging and cancer, resulting from the progressive accumulation of biochemical damage [6]. Therefore, CP-induced testicular

Received: Nov 13, 2018 Revised: Dec 15, 2018 Accepted: Dec 27, 2018 Published online Feb 7, 2019

Correspondence to: Cem Yucel (iD https://orcid.org/0000-0003-0838-9199

Department of Urology, Tepecik Training and Research Hospital, Yenişehir Mah, Gaziler Cad. No:468, Konak, İzmir, Turkey.

Tel: +90-5376676983, Fax: +90-2324330756, E-mail: meclecuy@hotmail.com 
toxicity can be eliminated with antioxidants.

Vitamin A and carotenoids have potent antioxidant effects [7]. Vitamin A is found as retinol, retinal, and retinoic acid in the body. Retinoic acid captures free radicals without interacting with biological targets and prevents free radical formation with its chain-breaking effect. Retinoic acid prevents lipid peroxidation in cellular membranes [8], and also has anti-proliferative, anti-inflammatory, and anti-cancer effects [9].

Although animal tests have been conducted with many antioxidant molecules to prevent CP-induced testicular toxicity, the effects of all-trans retinoic acid (ATRA) with and without CP have not been investigated. Here, we investigated the effects of ATRA use on CP-induced testicular toxicity in an experimental rat model.

\section{MATERIALS AND METHODS}

\section{Chemicals}

CP was purchased from Kocak Pharma (Istanbul, Turkey) and ATRA was purchased from Roche (Istanbul, Turkey).

\section{Animals}

A total of 28 male adult Wistar albino rats (age, 12 months old; weight, 270-320 g) were used. The rats were sheltered at constant room temperature $\left(23^{\circ} \mathrm{C}-\right.$ $24^{\circ} \mathrm{C}$ ) and relative humidity $(50 \%-55 \%)$ on a $12: 12$ hours light/dark cycle, in a well-ventilated room with clean plastic cages. These standard laboratory conditions were provided 7 days before commencement of the study. Water and feed were provided ad libitum. Feed and water were changed daily.

\section{Ethics statement}

All procedures were performed in accordance with the Guide for the Care and Use of Laboratory Animals of the Research Council after approval had been obtained from the Dokuz Eylul University (approval No. 02/22/2017-12; December 2017).

\section{Experimental design}

The animals were randomly divided into four groups of seven rats each. The rats in control group received a single-dose injection of saline $1 \mathrm{~mL} / \mathrm{kg}$ intraperitoneally (IP) for 10 days. The rats in ATRA group were injected with a single dose of ATRA $(7.5 \mathrm{mg} / \mathrm{kg})$ IP for
10 days. The rats in ATRA+CP group were injected with a single dose of ATRA $(7.5 \mathrm{mg} / \mathrm{kg})$ IP for 10 days and a single dose of $\mathrm{CP}(7 \mathrm{mg} / \mathrm{kg} \mathrm{IP})$ on day 4 . The rats in CP group were injected with a single dose of CP (7 $\mathrm{mg} / \mathrm{kg} \mathrm{IP}$ ) on day 4 of the 10-day therapy. ATRA was dissolved in pure corn oil, and no solvent was used for CP. The ATRA and CP doses as well as administration times were specified according to similar previous studies $[8,10,11]$.

\section{Sample collection}

The weights of all animals were measured on day 11, and they were sacrificed with a high dose of ether. Blood samples were collected via cardiac puncture. The abdomen was opened through a midline incision and a bilateral orchiectomy was rapidly performed. The testes were removed and weighed. The testis used for biochemical analyses was washed in cold saline solution twice and stored at $-80^{\circ} \mathrm{C}$ until subsequent analyses. The other was placed in formalin solution and held for histopathological analyses.

\section{Sperm analyses}

Epididymal sperm was counted with a hemocytometer using a modified method described by Yokoi et al [12]. The epididymis was minced finely using anatomical scissors in physiological saline $(5 \mathrm{~mL})$, placed in a rocker for 10 minutes, and incubated at room temperature for 2 minutes. The supernatant fluid was diluted 1:100 with a solution containing 5 g sodium bicarbonate, $1 \mathrm{~mL}$ formalin (35\%), and $25 \mathrm{mg}$ eosin per $100 \mathrm{~mL}$ distilled water. A $10 \mu \mathrm{L}$ aliquot of this diluted solution was placed in a sperm-counting chamber and analyzed by light microscopy under 400× magnification after 5 minutes.

Progressive sperm motility was determined using the method described by Sönmez et al [13]. Fluid was taken from the caudal epididymis using a pipette and diluted with $2 \mathrm{~mL}$ Tris buffer solution. The system was prewarmed $\left(35^{\circ} \mathrm{C}\right)$, three different fields were examined to determine sperm motility, and the mean value was recorded as the final motility score.

The slides were stained with eosin-nigrosin to determine the percentage of morphologically abnormal spermatozoa. A total of 300 sperm cells were examined on each slide under $400 \times$ magnification, and anomalies were expressed as percentages. 


\section{Biochemical analyses}

Testicular tissues were separated into small pieces after weighing. These tissues were diluted 10 -fold with $50 \mathrm{mM}$ phosphate-buffered saline solution and homogenized using a homogenizer (TissueLyser LT; Qiagen, Dublin, Ireland). The homogenate was centrifuged at 10,000 rpm for 15 minutes at $4^{\circ} \mathrm{C}$. The total antioxidant status (TAS) in testicular tissues was measured using an auto-analyzer (AU5800; Beckman Coulter Inc., Brea, CA, USA) and the Rel Assay kit (Rel Assay Diagnostics, Gaziantep, Turkey) following Erel [14]. This kit works according to the principle of blue-green 2,2'azino-bis (3-ethylbenzothiazoline-6-sulfonic acid) (ATBS) or the ABTS radical being reduced to its colorless form by antioxidant molecules. The change in absorbance at $660 \mathrm{~nm}$ is related to the total antioxidant level of the sample. Trolox, the water-soluble analogue of vitamin E, was used as the calibrator. The results are expressed as equivalent/g.

Total oxidant status (TOS) in testicular tissue was measured using the AU5800 auto-analyzer and the Rel Assay kit following Erel [15]. This kit works by the principle of oxidant molecules reducing the ferrous form of iron ions to the ferric form. The iron ions form a colorful complex with chromogen in an acidic environment. The color intensity measured with a spectrophotometer is related to the number of total oxidant molecules in the sample. Hydrogen peroxide was used as the calibrator, and the results are expressed as $\mu \mathrm{mol}$ $\mathrm{H}_{2} \mathrm{O}_{2}$ equivalent/g.

The oxidative stress index (OSI) is the ratio of TOS to TAS [15]. The results are expressed as an arbitrary unit (AU).

\section{Histopathological examination}

Testicular tissue was embedded in paraffin blocks after being fixed in 10\% formalin solution. Sections 4 $\mu \mathrm{m}$ thick were obtained, stained with $\mathrm{H} \& \mathrm{E}$, and examined under 400× magnification (CX31; Olympus, Tokyo, Japan). The severity of testicular damage was graded according to the presence of fibrosis: 0 , no fibrosis; 1 , fibrosis in $<25 \%$ of total testicular tissue (mild); 2 , fibrosis in $25 \%$ to $50 \%$ of total testicular tissue (moderate), and 3 , fibrosis $>50 \%$ of total testicular tissue (severe) [16].

Spermatogenesis in testicular tissue was determined using the Johnsen score (JS). In all, 100 seminiferous tubules were examined in each testis. The germinal epithelium of each tubule was scored between 1 and 10 according to tissue maturation and spermatogenesis status. Tubular sclerosis was scored as 1, only Sertoli cells as 2 , only spermatogonia as 3 , interruption at the primary spermatocyte stage as 4 or 5 , interruption at the early spermatid stage as 6 or 7 , interruption at the late spermatid stage as 8 or 9 , and full spermatogenesis as 10 [17].

\section{Statistical analyses}

We conducted power analyses before the study and found that seven rats per group was sufficient to interpret the results ( $86 \%$ actual power at $95 \%$ confidence interval with $\eta 2=0.35$ and effect size $f=0.73$ ). Categorical variables are expressed as numbers (n) and percentages (\%), and numerical variables are expressed as means and standard deviations if they fulfilled the assumptions of normality, and medians (range) if they did not. The normality of the distribution of numerical variables was tested using the Shapiro-Wilk test. Oneway analysis of variance was used if the difference between the groups met the parametric test assumptions, and Tukey's test was used for post hoc comparisons. The Kruskal-Wallis test was used if the differences among the groups did not meet the parametric test assumption, and the Dunn-Bonferroni test was used for post hoc comparisons.

The dependence between the categorical variables and the group was tested using the Fisher-FreemanHalton exact test.

Data analyses were performed using the IBM Statistical Package for the Social Science ver. 22.0 software (IBM Corp., Armonk, NY, USA), and a p-value <0.05 was considered statistically significant.

\section{RESULTS}

\section{Body weight and testis weight}

The loss in body weight of the rats was greater in the $\mathrm{CP}$ groups $(\mathrm{CP}$ and ATRA+CP) than in the control group $(p<0.01)$. A significant decrease was observed in the CP group compared to controls $(p<0.05)$. Similarly, testicular weight was significantly lower in the ATRA+CP group than in the control and ATRA groups $(p<0.05)$. Although testicular weight was higher in the ATRA+CP group than in the CP group, the difference was not significant (Table 1). 


\section{Sperm parameters}

Sperm count and motility significantly decreased in the CP group compared to the control and ATRA groups, and the percentage of abnormal sperm increased. Administering ATRA together with CP significantly increased sperm count and motility, and significantly decreased the percentage of abnormal sperm (Fig. 1). A comparison of the sperm parameters is given in Table 1.

\section{Biochemical evaluation}

Administering CP significantly increased TOS and the OSI $(p<0.05)$. By contrast, administering ATRA together with CP significantly decreased TOS and OSI in testicular tissue $(p<0.05)$. The OSI significantly increased in the ATRA+CP group compared to the ATRA group; however, no differences were found compared to the control group, and no significant differences were observed among groups with regard to TAS levels. A comparison of the groups with regard to TAS, TOS, and the OSI is presented in Table 2. Box plots showing the distribution of the TAS, TOS, and OSI levels in the groups are displayed in Fig. 2.

\section{Histopathological examination}

Normal testicular histopathology and spermatogenesis were observed in rats in the control and ATRA groups. The most evident changes in the $\mathrm{CP}$ group were maturational loss in germinal cells and arrest of spermatogenesis at the primary spermatocyte stage (Fig.

Table 1. Effect of all-trans retinoic acid on epididymal sperm characteristics

\begin{tabular}{lcccc}
\multicolumn{1}{c}{ Variable } & Control group & ATRA group & ATRA+CP group & CP group \\
\hline Testis weight & $0.142 \pm 0.15$ & $0.148 \pm 0.15$ & $0.116 \pm 0.12^{\mathrm{a}, \mathrm{b}}$ & $0.110 \pm 0.14^{\mathrm{a}, \mathrm{b}}$ \\
Sperm Count $\left(10^{6} / \mathrm{mL}\right)$ & $157(141-186)$ & $178(167-198)$ & $115(78-138)^{\mathrm{a}, \mathrm{b}}$ & $66(61-75)^{\mathrm{a}, \mathrm{b}, \mathrm{c}}$ \\
Sperm motility $(\%)$ & $77.43 \pm 6.26$ & $80.29 \pm 5.31$ & $57.14 \pm 5.20^{\mathrm{a}, \mathrm{b}}$ & $40.14 \pm 5.98^{\mathrm{a}, \mathrm{b}, \mathrm{c}}$ \\
Abnormal sperm (\%) & $7(6-11)$ & $7(5-9)$ & $12(8-14)^{\mathrm{a}, \mathrm{b}}$ & $16(12-23)^{\mathrm{a}, \mathrm{b}, \mathrm{c}}$ \\
\hline
\end{tabular}

Values are presented as mean \pm standard deviation or median (range).

ATRA: all-trans retinoic acid, CP: cisplatin.

${ }^{a} p<0.01$ compared with control group. ${ }^{b} p<0.01$ compared with ATRA group. ${ }^{c} p<0.01$ compared with ATRA+CP group.

A

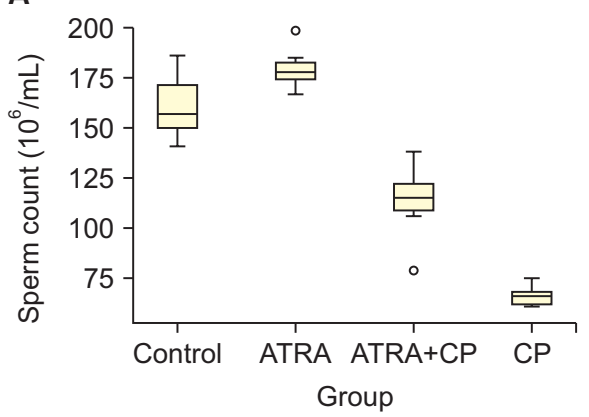

B

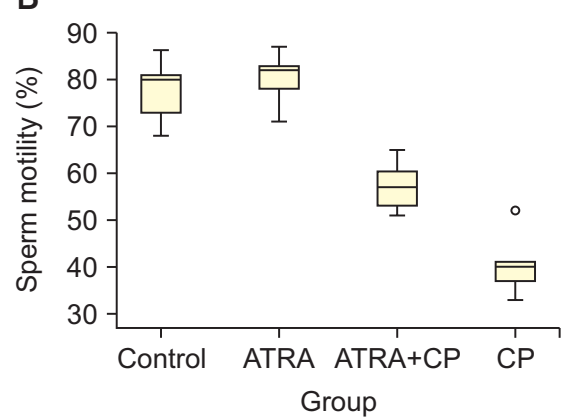

C

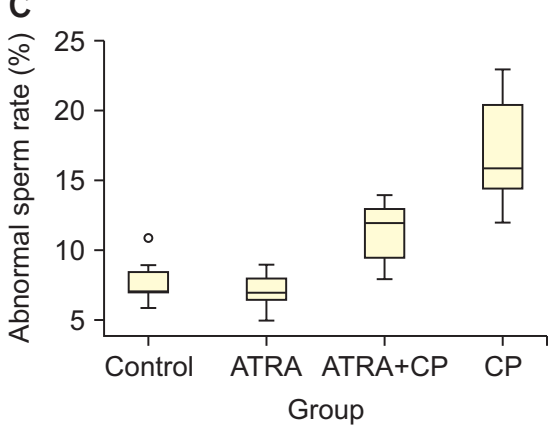

Fig. 1. Box graphics of sperm count (A), motility (B), and abnormal sperm rate (C). ATRA: all-trans retinoic acid, CP: cisplatin.

Table 2. Comparison of biochemical findings of groups

\begin{tabular}{lcccc}
\multicolumn{1}{c}{ Variable } & Control group & ATRA group & ATRA+CP group & CP group \\
\hline TAS $(\mu \mathrm{mol}$ Trolox equivalent/g) & $19.59 \pm 0.79$ & $20.67 \pm 0.74$ & $19.83 \pm 1.02$ & $20.55 \pm 1.67$ \\
TOS $\left(\mu \mathrm{mol} \mathrm{H}_{2} \mathrm{O}_{2}\right.$ equivalent/g) & $0.03(0.02-0.05)$ & $0.03(0.02-0.05)$ & $0.04(0.03-0.06)$ & $0.06(0.03-0.09)^{\mathrm{a}, \mathrm{b}, \mathrm{c}}$ \\
OSI & $0.15(0.09-0.25)$ & $0.13(0.09-0.24)$ & $0.18(0.14-0.29)^{\mathrm{b}}$ & $0.25(0-0.5)^{\mathrm{a}, \mathrm{b}, \mathrm{c}}$ \\
Testicular damage score & $0(0-0)$ & $0(0-0)$ & $1(0-2)$ & $2(1-2)^{\mathrm{a}, \mathrm{b}, \mathrm{c}}$ \\
Johnsen score & $10(10-10)$ & $10(10-10)$ & $8(6-8)^{\mathrm{a}, \mathrm{b}}$ & $5(4-6)^{\mathrm{a}, \mathrm{b}, \mathrm{c}}$ \\
\hline
\end{tabular}

Values are presented as mean \pm standard deviation or median (range).

ATRA: all-trans retinoic acid, CP: cisplatin, TAS: total antioxidant status, TOS: total oxidant status, OSI: oxidative stress index.

${ }^{a} \mathrm{p}<0.05$ compared with control group. ${ }^{b} p<0.05$ compared with ATRA group. ${ }^{c} p<0.05$ compared with ATRA+CP group. 
A

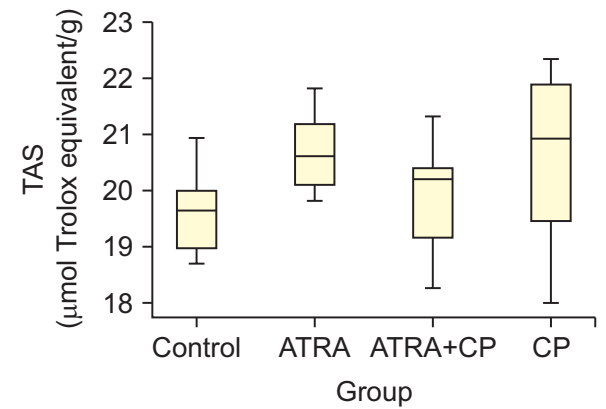

B

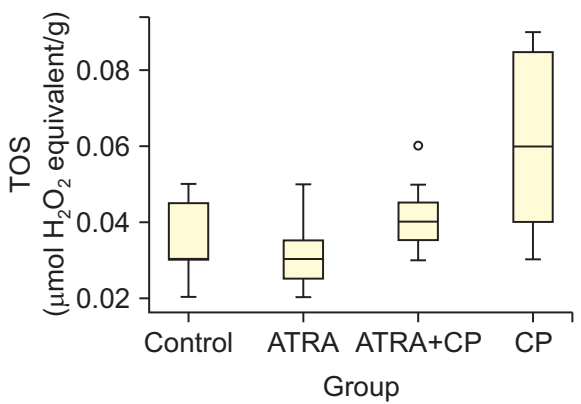

C

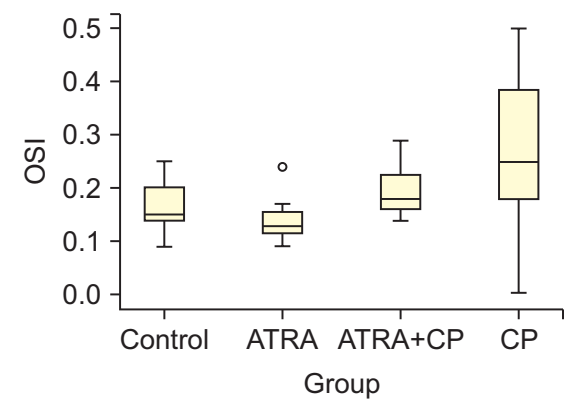

Fig. 2. Box graphics of total antioxidant status (TAS) (A), total oxidant status (TOS) (B), and oxidative stress index (OSI) (C). ATRA: all-trans retinoic acid, CP: cisplatin.
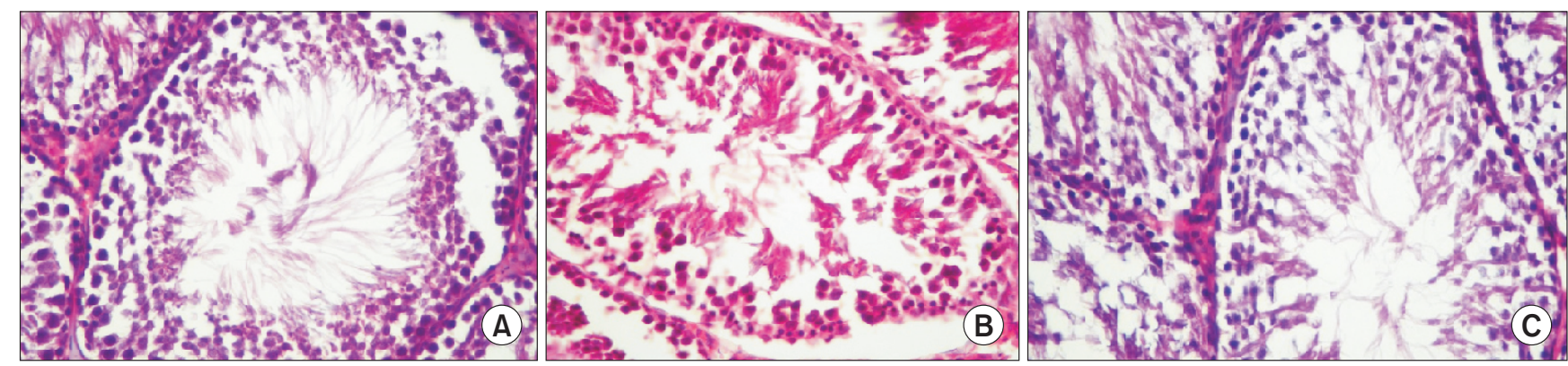

Fig. 3. Testicular histopathology in control group (A), all-trans retinoic acid+cisplatin (CP) group (B), and CP group (C) (A-C: H\&E, $\times 400)$.

3). Mild perivascular fibrosis, disorganization, and hyalinization of intertubular connective tissue were also observed. The testicular damage score was significantly higher in the CP group than in the control and ATRA groups. The JS was low due to early maturation arrest of spermatogenesis in the CP group. The most common histopathological condition was hypo-spermatogenesis due to mild degeneration in the CP+ATRA group. Administering ATRA together with CP decreased the arrest in spermatogenesis and increased the JS.

\section{DISCUSSION}

$\mathrm{CP}$ is an effective anti-neoplastic agent used to treat tumors such as those of the gallbladder, prostate, testicles, and other organs. However, its toxic effects restrict use of the drug. For example, $\mathrm{CP}$ can impair sperm quality and infertility due to its toxic effects on Sertoli, Leydig, and germ cells [18]. Therefore, it is import to prevent such side effects to preserve the fertilization capacity of patients who use CP. The results of this study indicate that using CP and ATRA together could reduce the destructive effects of $\mathrm{CP}$ on the testes. This is the first animal study to investigate the effects of ATRA on the toxic effects of CP.

Vitamin A converts into the ATRA active form via retinol and retinal dehydrogenase [19]. Previous studies have reported that spermatogenesis is arrested in rodents with retinoic acid insufficiency, which improves after administration of retinoic acid [20]. Vitamin A has effects on spermatogenesis via retinoic acid receptors [21], and genetic deletions in these receptors lead to sterility in rats [22].

Many studies have reported decreases in testis weight in rats administered CP $[1,2,6]$. By contrast, other studies have reported that $\mathrm{CP}$ treatment has no effect on testis weight $[18,23]$. This difference may be related to different sensitivities to the drug despite being administered at the same doses. Testis weight decreased in rats receiving $\mathrm{CP}$, which was related to structural disruption of the testis due to organ-specific toxicity by CP. Body weight also decreased due to malnutrition related to the toxic effects of CP.

CP-related testicular toxicity is associated with oxidative stress and ROS [3-5]. The main ROS include the superoxide anion, hydroxyl radical, and $\mathrm{H}_{2} \mathrm{O}_{2}$ [24]. ROSproducing pro-oxidants and ROS-scavenger antioxidant molecules are balanced in normal cells. Cellular dam- 
age related to toxic substances, such as $\mathrm{CP}$, disrupts this balance toward the oxidative side [3,25]. CP-related oxidative damage decreases in response to free radical scavengers and antioxidants, such as quercetin, chrysin, melatonin, vitamin $\mathrm{C}$, and vitamin $\mathrm{E}$ [26-28]. In our study, TOS and the OSI were used as indicators of pro-oxidant molecules. The TOS and the OSI values increased due to CP but significantly decreased after administration of ATRA, indicating that ATRA has positive effects on CP-related oxidative damage. No significant differences were detected among groups with regard to TAS values. The antioxidant molecules that increased to balance the elevated levels of pro-oxidant molecules in damaged testicular cells may have led to this result.

Administering CP decreased sperm count and motility and increased the production of abnormal sperm, consistent with many studies that have investigated the side effects of CP on sperm function [1,6,16,18]. Impaired sperm function is among the most important causes of infertility. ROS are produced by sperm cells, macrophages, and neutrophils in response to $\mathrm{CP}$ toxicity [16]. Spermatogenic cells are highly susceptible to ROS due to their high mitotic activity. Human studies have revealed that oxidative stress negatively affects sperm motility, vitality, and morphology [6]. ROS impair the permeability and viscosity function of the sperm membrane, leading to polyunsaturated fatty acid peroxidation [16]. CP-related toxic effects reduce ATP production by affecting the mitochondria of sperm cells. The decrease in ATP in sperm cells impairs flagellar function, leading to sperm immobility, DNA fragmentation in advanced stages, and apoptosis [16]. In this study, administering ATRA had positive effects on sperm count, motility, and morphology, which resulted from its antioxidant and free radical scavenging effects.

Administering CP leads to histopathological changes in the testes [3,4]. Consistent with the literature, we observed structural changes in testicular tissues. The most evident CP-related changes were maturation loss in germinal cells and spermatogenesis arrest of primary spermatocytes. Changes such as mild perivascular fibrosis, disorganization, and hyalinization of intertubular connective tissue were also observed. Administering ATRA with CP reduced the arrest in spermatogenesis and increased the JS. ATRA improved the disrupted testicular structure through its antioxi- dant effects, thereby demonstrating positive effects on spermatogenesis.

Previous studies have revealed that administering ATRA increases the anti-proliferative and pro-apoptotic effects of CP on cancer cells [29,30]. A rat experimental tumor model was used to investigate the effects of ATRA on preventing the toxic effects of CP. Our study may pioneer future similar rat studies, as the model was easy to apply and used TAS and TOS instead of many oxidant and antioxidant molecules concurrently. One limitation of our study was the lack of confirmation by immunohistochemical and electron microscopic findings.

\section{CONCLUSIONS}

The results of this study verify the destructive effects of CP treatment on testicular tissue and spermatogenesis. Administering ATRA with CP decreased oxidative stress in testicular cells and reversed spermatogenesis and histopathologic impairments. These beneficial effects of ATRA likely result from its antioxidant features. More studies should be conducted before ATRA is used clinically to preserve fertility in patients receiving $\mathrm{CP}$ treatment.

\section{ACKNOWLEDGEMENTS}

We would like to thank to Pelin Teke Kisa, Serdar Bayrak and Ozgur Cakmak for their contribution to the statistical analysis.

\section{Disclosure}

The authors have no potential conflicts of interest to disclose.

\section{Author Contribution}

Conceptualization: Yucel C. Data curation: Arslan FD, Ekmekci S. Formal analysis: Ulker V, Kisa E. Funding acquisition: None. Investigation: Yucel C, Ulker V. Methodology: Ucar M, Yucel EE. Project administration: Ilbey YO, Celik O. Resources: Yucel C, Basok BI. Software: Kozacioglu Z. Supervision: Basok BI, Kozacioglu Z. Validation: Yucel C. Visualization: Yucel C, Ulker V. Writing-original draft: Yucel C. Writingreview \& editing: Yucel C, Yucel EE. 


\section{Data Sharing Statement}

The data required to reproduce these findings cannot be shared at this time due to legal and ethical reasons.

\section{REFERENCES}

1. Adejuwon SA, Femi-Akinlosotu OM, Omirinde JO. Cisplatin-induced testicular dysfunction and its amelioration by Launaea taraxacifolia leaf extract. Andrologia 2015;47:553-9.

2. Ilbey YO, Ozbek E, Cekmen M, Simsek A, Otunctemur A, Somay A. Protective effect of curcumin in cisplatin-induced oxidative injury in rat testis: mitogen-activated protein kinase and nuclear factor-kappa B signaling pathways. Human Reprod 2009;24:1717-25.

3. Ilbey YO, Ozbek E, Simsek A, Cekmen M, Otunctemur A, Somay A. Chemoprotective effect of a nuclear factor-kappaB inhibitor, pyrrolidine dithiocarbamate, against cisplatininduced testicular damage in rats. J Androl 2009;30:505-14.

4. Ciftci O, Cetin A, Aydin M, Kaya K, Oguz F. Fish oil, contained in eicosapentaenoic acid and docosahexaenoic acid, attenuates testicular and spermatological damage induced by cisplatin in rats. Andrologia 2014;46:1161-8.

5. Tuglu D, Yuvanc E, Yilmaz E, Gencay IY, Atasoy P, Kisa U, et al. The antioxidant effect of dexmedetomidine on testicular ischemia-reperfusion injury. Acta Cir Bras 2015;30:414-21.

6. Salem EA, Salem NA, Maarouf AM, Serefoglu EC, Hellstrom WJ. Selenium and lycopene attenuate cisplatin-induced testicular toxicity associated with oxidative stress in Wistar rats. Urology 2012;79:1184.

7. Amory JK, Ostrowski KA, Gannon JR, Berkseth K, Stevison $\mathrm{F}$, Isoherranen $\mathrm{N}$, et al. Isotretinoin administration improves sperm production in men with infertility from oligoasthenozoospermia: a pilot study. Andrology 2017;5:1115-23.

8. Elsayed AM, Abdelghany TM, Akool el-S, Abdel-Aziz AA, Abdel-Bakky MS. All-trans retinoic acid potentiates cisplatininduced kidney injury in rats: impact of retinoic acid signaling pathway. Naunyn Schmiedebergs Arch Pharmacol 2016; 389:327-37.

9. Zuno-Floriano FG, Holstege D, Hengel MJ, Gaikwad NW, Aldana-Madrid ML, Miller MG. Determination of vitamin A and its metabolites in rat testis: possible involvement of vitamin A in testicular toxicity caused by molinate. Bull Environ Contam Toxicol 2012;88:1038-42.

10. Rao J, Zhang C, Wang P, Lu L, Zhang F. All-trans retinoic acid alleviates hepatic ischemia/reperfusion injury by enhancing manganese superoxide dismutase in rats. Biol Pharm Bull 2010;33:869-75.
11. Ewees MG, Abdelghany TM, Abdel-Aziz AA, Abdel-Bakky MS. All-trans retinoic acid mitigates methotrexate-induced liver injury in rats; relevance of retinoic acid signaling pathway. Naunyn Schmiedebergs Arch Pharmacol 2015;388:9318.

12. Yokoi K, Uthus EO, Nielsen FH. Nickel deficiency diminishes sperm quantity and movement in rats. Biol Trace Elem Res 2003;93:141-54.

13. Sönmez M, Türk G, Yüce A. The effect of ascorbic acid supplementation on sperm quality, lipid peroxidation and testosterone levels of male Wistar rats. Theriogenology 2005;63: 2063-72.

14. Erel O. A novel automated method to measure total antioxidant response against potent free radical reactions. Clin Biochem 2004;37:112-9.

15. Erel O. A new automated colorimetric method for measuring total oxidant status. Clin Biochem 2005;38:1103-11.

16. Ilbey YO, Ozbek E, Simsek A, Otunctemur A, Cekmen M, Somay A. Potential chemoprotective effect of melatonin in cyclophosphamide- and cisplatin-induced testicular damage in rats. Fertil Steril 2009;92:1124-32.

17. Johnson L, Petty CS, Neaves WB. The relationship of biopsy evaluations and testicular measurements to over-all daily sperm production in human testes. Fertil Steril 1980;34:3640.

18. Kaya K, Ciftci O, Cetin A, Doğan H, Başak N. Hesperidin protects testicular and spermatological damages induced by cisplatin in rats. Andrologia 2015;47:793-800.

19. van Beek ME, Meistrich ML. Spermatogenesis in retinol-deficient rats maintained on retinoic acid. J Reprod Fertil 1992; 94:327-36.

20. Chung SS, Wolgemuth DJ. Role of retinoid signaling in the regulation of spermatogenesis. Cytogenet Genome Res 2004; 105:189-202.

21. Hogarth CA, Griswold MD. The key role of vitamin A in spermatogenesis. J Clin Invest 2010;120:956-62.

22. van Pelt AM, de Rooij DG. Synchronization of the seminiferous epithelium after vitamin A replacement in vitamin Adeficient mice. Biol Reprod 1990;43:363-7.

23. Beytur A, Ciftci O, Oguz F, Oguzturk H, Yilmaz F. Montelukast attenuates side effects of cisplatin including testicular, spermatological, and hormonal damage in male rats. Cancer Chemother Pharmacol 2012;69:207-13.

24. Sikka SC. Relative impact of oxidative stress on male reproductive function. Curr Med Chem 2001;8:851-62.

25. Choudhury RC, Jagdale MB. Vitamin E protection from/ potentiation of the cytogenetic toxicity of cisplatin in Swiss mice. J Chemother 2002;14:397-405. 
26. Giri A, Khynriam D, Prasad SB. Vitamin C mediated protection on cisplatin induced mutagenicity in mice. Mutat Res 1998;421:139-48.

27. Koksal M, Oğuz E, Baba F, Eren MA, Ciftci H, Demir ME, et al. Effects of melatonin on testis histology, oxidative stress and spermatogenesis after experimental testis ischemiareperfusion in rats. Eur Rev Med Pharmacol Sci 2012;16:5828.

28. Sherif IO, Abdel-Aziz A, Sarhan OM. Cisplatin-induced tes- ticular toxicity in rats: the protective effect of arjunolic acid. J Biochem Mol Toxicol 2014;28:515-21.

29. Moreb JS, Ucar-Bilyeu DA, Khan A. Use of retinoic acid/aldehyde dehydrogenase pathway as potential targeted therapy against cancer stem cells. Cancer Chemother Pharmacol 2017;79:295-301.

30. Tang XH, Gudas LJ. Retinoids, retinoic acid receptors, and cancer. Annu Rev Pathol 2011;6:345-64. 